Research Paper

\title{
Trefoil Factor I in Early Breast Carcinoma: A Potential Indicator of Clinical Outcome during the First 3 Years of Follow-Up
}

\author{
Milan Markićević́ ${ }^{\bowtie}$, Radan Džodić2,3, Marko Buta², Ksenija Kanjer¹, Vesna Mandušić4 ${ }^{4}$ Zora

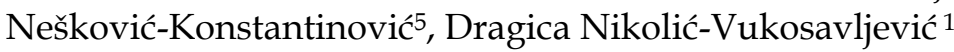 \\ 1. Department of Experimental Oncology, Institute for Oncology and Radiology of Serbia, Pasterova 14, 11000 Belgrade, Serbia; \\ 2. Surgical Oncology Clinic, Institute for Oncology and Radiology of Serbia, Pasterova 14, 11000 Belgrade, Serbia; \\ 3. University of Belgrade School of Medicine, Dr Subotića 8, 11000 Belgrade, Serbia; \\ 4. Vinča Institute of Nuclear Science, Mike Petrovića Alasa 12-14, 11000 Belgrade, Serbia; \\ 5. Clinic of Medical Oncology, Institute for Oncology and Radiology of Serbia, Pasterova 14, 11000 Belgrade, Serbia.
}

$\square$ Corresponding author: Milan Markićević, Department of Experimental Oncology, Institute for Oncology and Radiology of Serbia, Pasterova 14, 11000 Belgrade, Serbia, markicevic@ncrc.ac.rs.

(c) Ivyspring International Publisher. This is an open-access article distributed under the terms of the Creative Commons License (http://creativecommons.org/ licenses/by-nc-nd/3.0/). Reproduction is permitted for personal, noncommercial use, provided that the article is in whole, unmodified, and properly cited.

Received: 2013.11.22; Accepted: 2014.03.15; Published: 2014.05.01

\begin{abstract}
Background. A role of an estrogen-regulated, autocrine motogenic factor was assumed to be a major biological role of trefoil factor I (TFFI) in breast cancer. TFFI is regarded as a predictive factor for positive response to endocrine therapy in breast cancer patients. The aim of our study was to examine TFFI level distribution in breast carcinomas in order to distinguish estrogen-independent from estrogen-dependent TFFI expression and to evaluate clinical usefulness of TFFI status in early breast cancer during the first 3 years of follow-up.

Methods. The study included 226 patients with primary operable invasive early breast carcinomas for whom an equal, a 3-year follow-up was conducted. TFFI levels as well as estrogen receptor $(E R)$ and progesterone receptor (PR) levels were measured in cytosolic extracts of tumor samples by immunoradiometric assay or by use of classical biochemical method, respectively. Non-parametric statistical tests were applied for data analyses.

Results. Statistical analysis revealed that TFFI levels were significantly higher in premenopausal patients $(p=0.02)$, or in tumors with: lower histological grade $(p<0.001)$, positive ER or PR status $(p<0.00 \mathrm{I}$, in both cases). On the basis of TFFI level distribution between ER-negative and ER-positive postmenopausal patients with tumors of different histological grade, $14 \mathrm{ng} / \mathrm{mg}$ was set as the cut-off value to distinguish estrogen-independent from estrogen-dependent TFFI expression in breast cancer. Depending on menopausal and PR status, positive TFFI status identified patients at opposite risk for relapse among ER-positive patients with grade II tumors. Among ERand PR-positive premenopausal patients with grade II tumors, TFFI status alone identified patients at opposite risk for relapse.

Conclusions. Determination of TFFI status might identify patients at different risk for relapse and help in making decision on administering adjuvant therapy for early breast cancer patients during the first 3 years of follow-up.
\end{abstract}

Key words: trefoil factor 1; cut-off value, disease-free interval; early breast carcinoma; early follow-up.

\section{Introduction}

Trefoil factors (TFFs) represent a family of small peptides $(6-10 \mathrm{kDa})$ characterized by a common se- quence, consisting of 42-43 amino-acids and highly conserved in mammals [1]. Intramolecular disulphide 
bonds within the sequence cause the formation of the characteristic three-loop structure, called the trefoil domain [1, 2], making TFFs stable molecules that are relatively resistant to proteases. Three mammalian trefoil peptides are known: TFF1 (formerly pS2) and TFF3 (intestinal trefoil peptide) contain one while TFF2 (spasmolytic polypeptide) contains two trefoil domains [3]. Trefoil factor 1 is a small cysteine-rich acidic peptide consisting of 60 amino acids [1]. In normal human tissues, TFF1 is predominantly expressed in the gastrointestinal tract [4] and, although its biological function is not clarified, it seems to have an important role in preserving the mucosa of the gastrointestinal tract [5]. In malignant tissue, TFF1 was initially found in breast cancer [6], but it has also been detected in several other carcinomas, such as stomach, pancreas, large intestine, endometrium, ovary, uterus, bladder, and prostate [7-10].

Hormones (estradiol [11]), growth factors such as insulin-like growth factor I (IGF-I), IGF-II, epidermal growth factor (EGF) or transforming growth factor (TGF) [11-13], phorbol esters [11, 14], plasminogen activator and Fos and Jun oncoproteins [11] have been reported to regulate the expression of the TFF1 through the activation of the gene promoter containing an estrogen-response element (ERE) and a TPA-response element (TRE) [15]. The peptide was found to be overexpressed in approximately $50 \%$ of primary breast carcinomas [16], mainly estrogen receptor alpha-positive $(E R \alpha+)$ ones. It has been demonstrated that estrogen stimulation of an estrogen-dependent breast cancer cell line induce significant (up to 100-fold) increase of TFF1 mRNA as well as an increase of protein level [6].

Despite numerous studies, the biological function of TFF1 in breast cancer is not yet clarified. Considering that low levels of TFF1 were shown to be characteristic for normal breast tissue, it has been proposed that overexpression of the peptide in breast cancer indicates its adverse function, possible as an oncogene. Early studies indicated that TFF1 might be a mitogen but they failed to demonstrate such biological function. Recent studies provided evidence regarding the possible role of TFF1 in breast cancer contributing to tumor aggressiveness. TFF1 was found to stimulate cell proliferation both in vitro and in models [17]. It has been reported that TFF1, induced by estrogen, stimulates migration of breast cancer cells $[18,19]$. Moreover, TFF1 dimer was shown to be more effective than TFF1 monomer in stimulation of breast cancer cell motility [19], possible due to its interaction with a putative cell surface receptor that has not been yet identified. A role of an estrogen-regulated, autocrine motogenic factor was assumed to be a major biological role of TFF1 in breast cancer.

The finding that TFF1 overexpression has been detected in approximately $50 \%$ of breast carcinomas [7], while only low levels of the peptide have been found in normal breast tissue [16] provided a rationale for numerous studies evaluating the potential clinical significance of TFF1 in breast cancer. The protein is not only regarded as an indicator of the intact ER signaling pathway [20,21], it is considered to be a predictive factor for positive response to endocrine therapy in breast cancer patients [21-26]. Furthermore, some studies suggested that TFF1 expression might be useful in identifying the subgroup of ER-positive breast cancer patients being more responsive to aromatase inhibitors (AI) than to Tamoxifen [27]. Most studies reported on association between TFF1 expression in breast cancer and a favorable prognosis [16, 21, 25, 28-32] while some studies failed to confirm such correlation [33].

In our study, a significant difference in TFF1 levels was found in relation to either menopausal status, histological grade, estrogen receptor (ER) status or progesterone receptor (PR) status. The first aim of our study was to determine a biologically-related cut-off value for TFF1 expression that would allow us to distinguish between estrogen-dependent and estrogen-independent TFF1 expression in breast cancer. On the basis of TFF1 level distribution in both premenopausal and postmenopausal patients bearing ER-positive and ER-negative tumors (regardless of histological grade), a concentration of $14 \mathrm{ng} / \mathrm{mg}$ of TFF1 was established as a cut-off value for TFF1 expression in breast cancer. The second aim of our study was to evaluate a potential clinical relevance of TFF1 in breast cancer, on the basis of the established cut-off value. Therefore, we analyzed disease free-interval (DFI) probabilities in relation to TFF1 status. TFF1 status allowed us to discriminate between patients with high versus low risk for development of distant metastases during a 3-year follow-up after the surgery.

\section{Methods}

This retrospective study included tumor samples from 226 consecutive female patients with clinical stage I or stage II breast cancer. After the surgery, primary operable invasive early breast carcinomas were histologically verified for all patients in the study. In the adjuvant setting of these patients, treated at the Institute for Oncology and Radiology of Serbia between 2002 and 2005, chemotherapy was given to 64 patients $(32 \%)$, the same number of patients received hormonotherapy, both types of systemic therapy were administered to 32 patients (16\%) while 41 patients $(20 \%)$ received no adjuvant therapy. Histo- 
logical specimens were reviewed and then classified according to the criteria of the International Union Against Cancer for TN stages [34] and according to the criteria for histological type [35] and grade [36]. Patients were between 24 and 78 years old (median, 56 years). Patients were considered to be postmenopausal if menstruation ceased for at least six months, otherwise they were considered as premenopausal. Patients' and tumor characteristics are presented in Table 1.

Table I. Cytosolic TFFIlevels in 226 breast carcinomas: correlation with different clinico-pathological parameters.

\begin{tabular}{|c|c|c|c|c|c|}
\hline \multirow{2}{*}{$\begin{array}{l}\text { Patient and tumor char- } \\
\text { acteristics }\end{array}$} & \multirow[t]{2}{*}{$\mathbf{n}$} & \multirow[t]{2}{*}{$\%$} & \multicolumn{2}{|c|}{ TFF1 (ng/mg) } & \multirow{2}{*}{$\begin{array}{l}\mathrm{p} \\
\text { value }\end{array}$} \\
\hline & & & Range & Median & \\
\hline Total & 226 & 100 & $1.5-135.8$ & 9 & \\
\hline \multicolumn{6}{|l|}{ Age (years) } \\
\hline$<45$ & 39 & 17.2 & $2-82.9$ & 17.7 & NS \\
\hline $45-59$ & 96 & 42.5 & $1.5-124.1$ & 7.9 & \\
\hline$>59$ & 91 & 40.3 & $1.8-135.8$ & 9.4 & \\
\hline \multicolumn{6}{|l|}{ Menopausal status } \\
\hline Premenopausal & 77 & 34.1 & $2-124.1$ & 13.6 & 0.02 \\
\hline Postmenopausal & 144 & 63.7 & $1.5-135.8$ & 8 & \\
\hline Unknown & 5 & 2.2 & & & \\
\hline \multicolumn{6}{|l|}{ Nodal status } \\
\hline pN0 & 92 & 40.7 & $1.5-135.8$ & 7.4 & NS \\
\hline $\mathrm{pN}+$ & 112 & 49.6 & $2-124.1$ & 11.2 & \\
\hline Unknown & 22 & 9.7 & $1.6-58.1$ & & \\
\hline \multicolumn{6}{|l|}{ Tumor size } \\
\hline pT1 & 100 & 44.2 & $1.5-135.8$ & 10.6 & NS \\
\hline pT2 & 114 & 50.4 & $1.9-124.6$ & 8.4 & \\
\hline pT3 & 8 & 3.6 & $2.2-19.4$ & 13.2 & \\
\hline Unknown & 4 & 1.8 & & & \\
\hline \multicolumn{6}{|l|}{ Histological grade } \\
\hline I & 33 & 14.6 & $1.9-56.2$ & 7.3 & $<0.001$ \\
\hline II & 146 & 64.6 & $1.6-124.6$ & 12.1 & \\
\hline III & 43 & 19 & $1.5-137.4$ & 3.1 & \\
\hline Unknown & 4 & 1.8 & & & \\
\hline \multicolumn{6}{|l|}{ Histological type } \\
\hline IDC & 112 & 49.6 & $1.5-137.4$ & 6.4 & NS \\
\hline ILC & 80 & 35.4 & $2-124.1$ & 12 & \\
\hline Other & 34 & 15 & $1.6-55.4$ & 13.7 & \\
\hline \multicolumn{6}{|l|}{ ER status } \\
\hline ER+ & 153 & 67.7 & $1.6-137.4$ & 15.9 & $<0.001$ \\
\hline ER- & 73 & 32.3 & $1.5-66$ & 3.7 & \\
\hline \multicolumn{6}{|l|}{ PR status } \\
\hline $\mathrm{PR}+$ & 102 & 45.1 & $1.6-137.4$ & 15.4 & $<0.001$ \\
\hline PR- & 124 & 54.9 & $1.5-124.6$ & 5.2 & \\
\hline
\end{tabular}

"Mann-Whitney or Kruskal-Wallis test.

Patients' follow-up was conducted every three months for the first two years and every six months during the third year. The recurrence of the disease was set as an end-point in our study because it is clinically useful in regard to metastatic disease progression. Disease-free interval was defined as time from the initial diagnosis to the emergence of distant metastases. The follow-up data were available for 201 patients and these patients were included in the survival analysis. Forty-three (21.4 \%) patients developed distant metastases during a 3-year follow-up. The study was approved by the Ethics Committee of the Institute of Oncology and Radiology of Serbia and the patients provided their informed consent before entering the study.

Estrogen receptor and progesterone receptor levels in cytosol extracts of tumor samples were measured by using the classical biochemical method as recommended by the European Organisation for Research and Treatment of Cancer (EORTC) [37]. The intra-laboratory quality assessment of the steroid hormone receptor levels was performed periodically following the EORTC recommendation [38]. The cut-off value for the quantitative classification of positive receptor status was $10 \mathrm{fmol} / \mathrm{mg}$ for ER and 20 $\mathrm{fmol} / \mathrm{mg}$ for PR [39]. TFF1 levels were assayed in tumor cytosols obtained for routine ER and PR determination and were measured by using a solid-phase radioimmunoassay (ELSA-pS2 Cis Biointernational, Gif-Sur-Yvet, France). The assay was performed according to the manufacturer's instructions. Concentration of TFF1 was expressed in $\mathrm{ng} / \mathrm{mg}$ protein. Cytosol protein concentration was determined by the Lowry method [40].

The non-parametric statistical methods were applied for data analyses. The distribution of TFF1 levels between the subgroups of patients was assessed using Mann-Whitney or Kruskal-Wallis test. Spearman's rank correlation test was used to assess the correlation between two variables. Survival curves for DFI were estimated according to the method of Kaplan and Meier. The log-rank test was used to compare survival functions. The distribution of different therapies between subgroups of patients was evaluated by Chi-squared test. A limit for significance was set at $\mathrm{p}<0.05$.

\section{Results}

The distribution of TFF1 levels within the analyzed group of 226 breast carcinomas was statistically different from the normal distribution (Kolmogorov-Smirnov test, $\mathrm{p}<0.001$ ) and it is presented in Figure 1. A wide range of TFF1 levels was detected in breast carcinomas and protein levels varied from 1.5 to $135.8 \mathrm{ng} / \mathrm{mg}$ protein, with a median value of $9 \mathrm{ng} / \mathrm{mg}$ protein. The distribution of TFF1 levels in relation to tumor-host (age, menopausal status) and tumor parameters (axillary node status, tumor size, histological grade, histological type, ER and PR status) is shown in Table 1. Statistical analysis revealed that TFF1 levels were significantly higher in premenopausal than in postmenopausal patients $(\mathrm{p}=0.02)$ as well as in patients bearing his- 
tological grade I or II tumors compared to those bearing histological grade III tumors $(p<0.001)$. In addition, ER-positive (ER+) or PR-positive (PR+) tumors were characterized with higher TFF1 levels in comparison to ER-negative (ER-) or PR-negative (PR-) tumors, respectively ( $\mathrm{p}<0.001$ in both cases). The last result may be expected, considering a statistically significant correlation between TFF1 and either ER or PR levels in analyzed samples of breast carcinoma $(\mathrm{r}=0.295, \mathrm{p}<0.001$ and $\mathrm{r}=0.254, \mathrm{p}<0.001$, respectively). Regardless of significant differences in TFF1 levels between the indicated groups of patients, the ranges of peptide levels between corresponding groups were comparable (as shown in Table 1) and we were unable, on the basis of these results, to define biologically-related cut-off value for TFF1 expression in breast carcinomas.

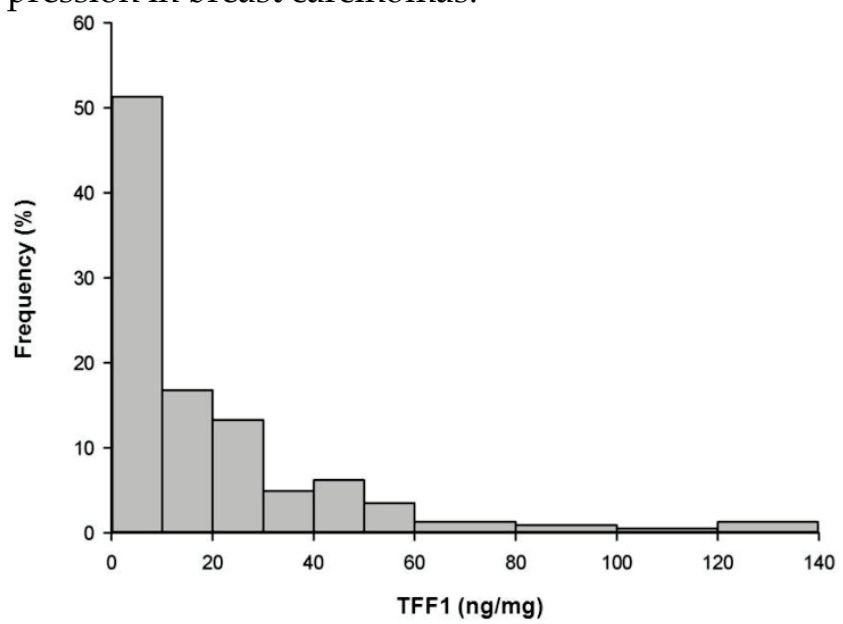

Figure I. Frequencies of cytosolic TFFI levels. Frequencies of cytosolic TFFI levels in 226 breast carcinomas, at intervals of $10 / 20 \mathrm{ng} / \mathrm{mg}$.
In an effort to discriminate estrogenindependent from estrogen-dependent TFF1 expression, the distribution of TFF1 levels was, further, analyzed between the groups of patients defined by combining menopausal status, histological grade, ER status and PR status. Figure 2 shows the distribution of TFF1 levels in ER-positive and ER-negative postmenopausal patients bearing tumors of different histological grade. Among postmenopausal patients, those with ER-negative status had significantly lower TFF1 levels in comparison to those with ER-positive status, irrespective of tumor histological grade ( $p=0.04, p<0.001$ and $p<0.05$ for histological grade I, II and III, respectively). On the other hand, the ranges of TFF1 levels of postmenopausal patients with tumors of different ER status (ER+ vs. ER-) were not comparable within the same histological grade of tumor. Since the highest TFF1 levels in ER-negative tumors of postmenopausal patients were similar across tumors of different histological grade (12.3, 13.9 and 9.6 for histological grades I, II and III, respectively), we established $14 \mathrm{ng} / \mathrm{mg}$ as the cut-off value to discriminate between estrogen-dependent and estrogen-independent TFF1 expression in breast cancer. Accordingly, we were able to define TFF1 status as a positive (TFF1+) or a negative one (TFF1-), depending on whether TFF1 levels in tumors were higher or lower than the cut-off value.

\footnotetext{
$\longrightarrow$
}

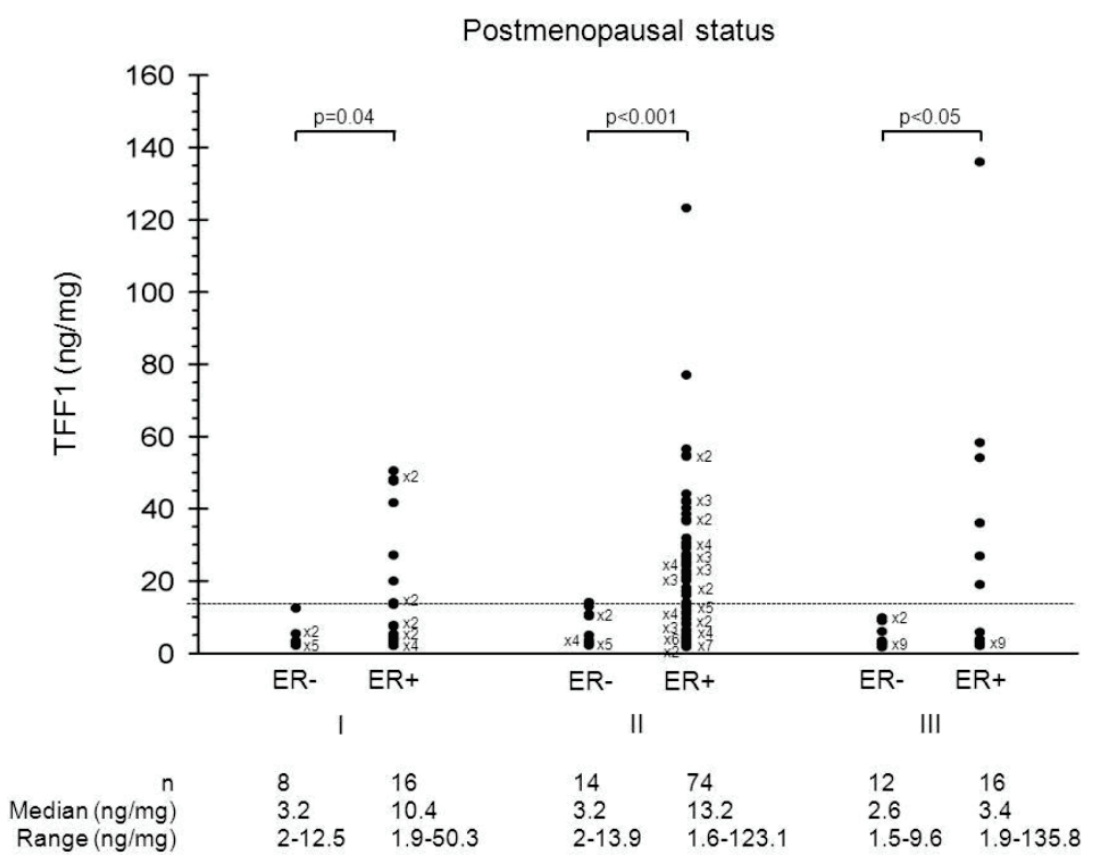

Figure 2. Distribution of TFFI levels. Distribution of TFFI levels in ER-positive and ER-negative postmenopausal patients bearing tumors of different histological grade. 
Statistical analysis showed that TFF1 status alone did not show a significant association with DFI probabilities during the first 3 years of patients' follow-up (data not shown). Therefore, we compared DFI probabilities between groups of patients defined by different clinico-pathological parameters, focusing to parameters that appeared to affect TFF1 level distribution in breast cancer.

Analysis of premenopausal patients bearing ER-positive and PR-positive tumors of histological grade II revealed a statistically significant difference in DFI probabilities between patients with positiveand negative TFF1 status, where those with positive TFF1 status had significantly lower DFI probabilities $(p<0.05$, Figure 3$)$. In addition, postmenopausal and

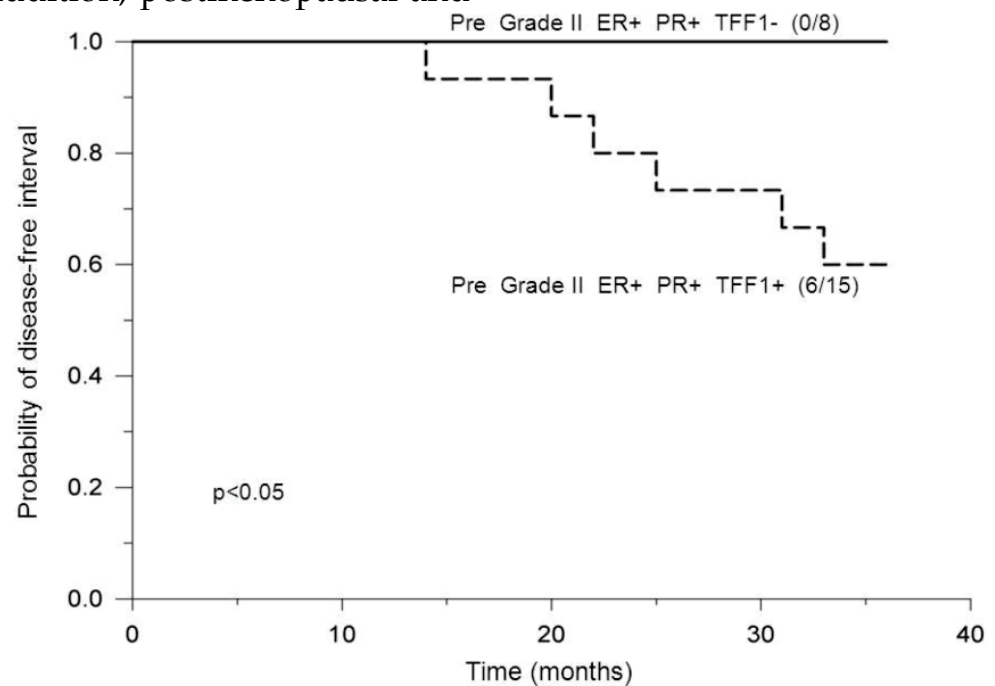

Figure 3. Probability of disease-free interval, stratified according to negative or positive TFFI status. Probability of disease-free interval for ER-positive and PR-positive premenopausal breast cancer patients bearing tumors of histological grade II, stratified according to negative (TFFI-) or positive (TFFI+) TFFI status. The number of patients with distant metastases/total number of patients is indicated in brackets. The solid line indicates negative and the dashed line indicates positive TFFI status. Pre: Premenopausal status.

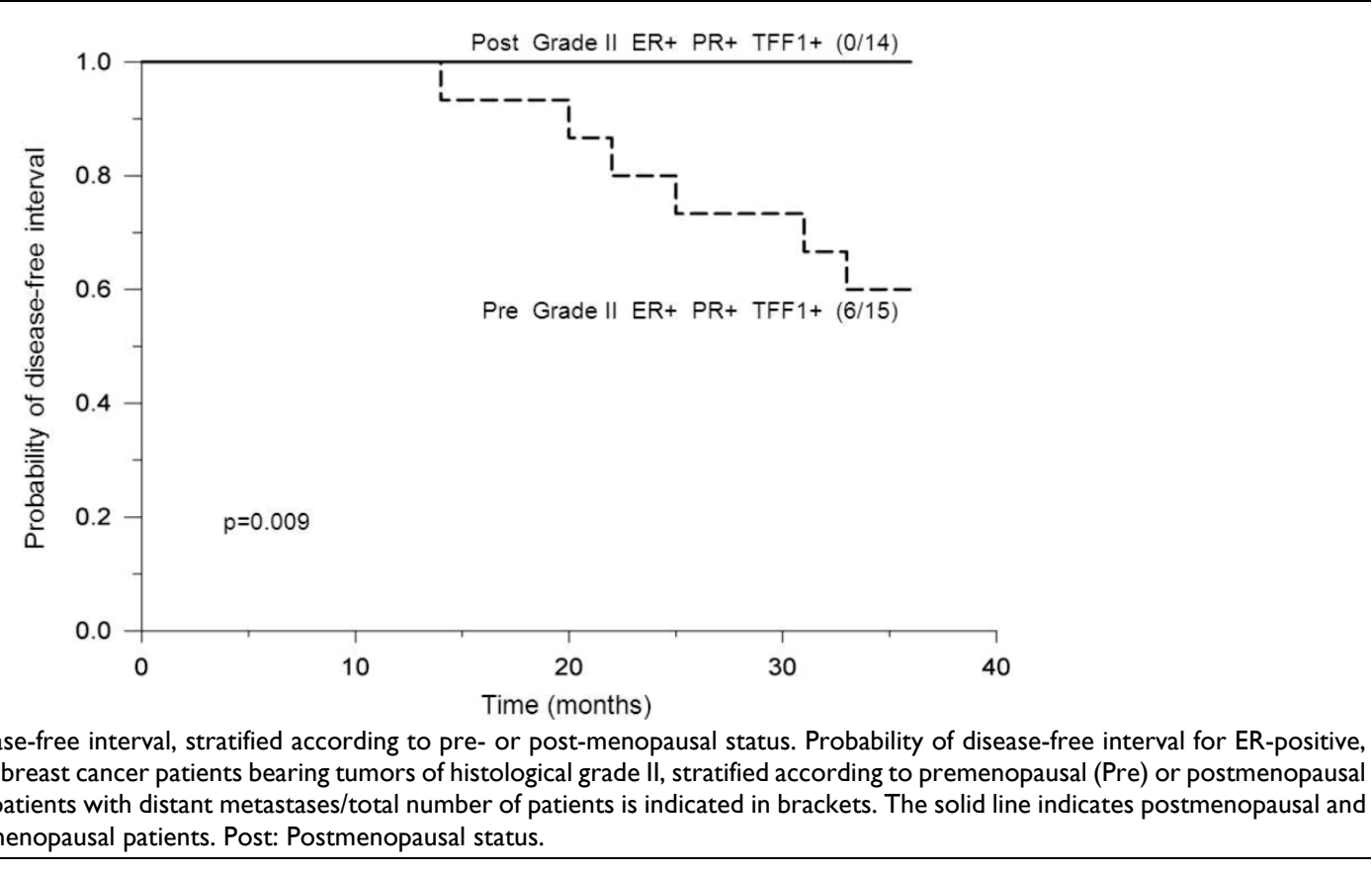

Figure 4. Probability of disease-free interval, stratified according to pre- or post-menopausal status. Probability of disease-free interval for ER-positive, PR-positive and TFFI-positive breast cancer patients bearing tumors of histological grade II, stratified according to premenopausal (Pre) or postmenopausal (Post) status. The number of patients with distant metastases/total number of patients is indicated in brackets. The solid line indicates postmenopausal and the dashed line indicates premenopausal patients. Post: Postmenopausal status.
PR status were found to affect DFI probabilities of TFF1-positive patients with ER-positive tumors of grade II in such a way that: a) among patients with positive PR status, premenopausal patients had significantly lower DFI probabilities in comparison to postmenopausal ones $(p=0.009$, Figure 4$)$ and $b)$ among postmenopausal patients, those with negative PR status had significantly lower DFI probabilities in comparison to those with positive PR status $(p=0.04$, Figure 5). By use of Chi-squared test, analysis revealed no difference in distribution of adjuvant systemic therapies among analyzed subgroups of patients during the first 3 years of follow-up (data not shown). 


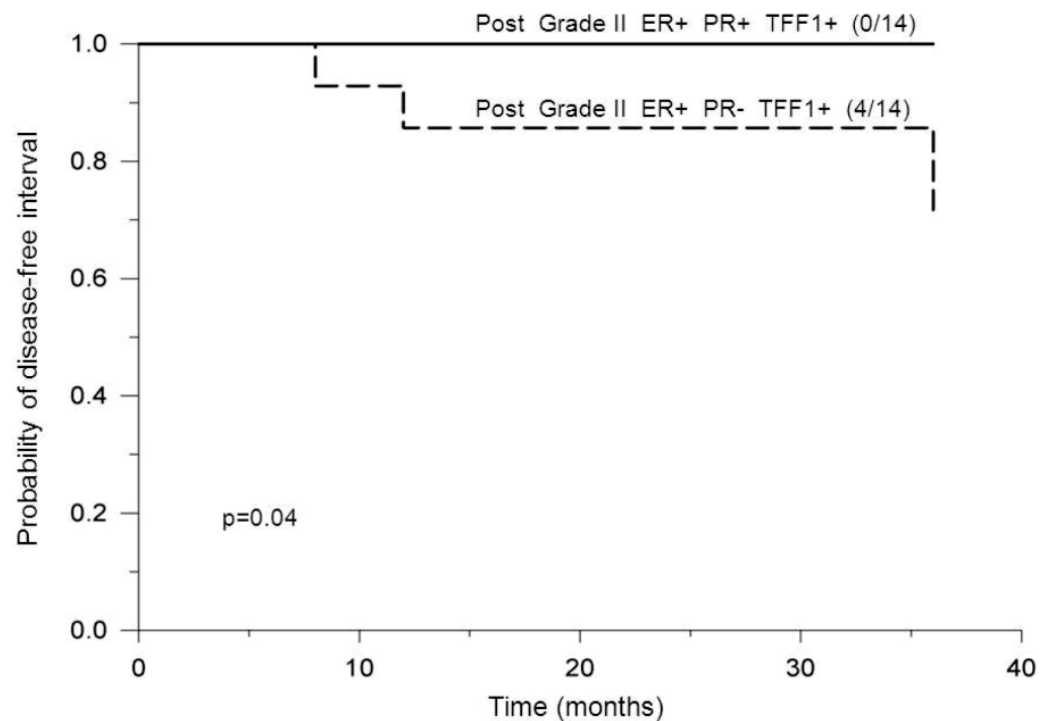

Figure 5. Probability of disease-free interval, stratified according to negative or positive PR status. Probability of disease-free interval for ER-positive and TFFI-positive postmenopausal breast cancer patients bearing tumors of histological grade II, stratified according to PR-negative (PR-) or PR-positive (PR+) status. The number of patients with distant metastases/total number of patients is indicated in brackets. The solid line indicates positive and the dashed line indicates negative PR status.

\section{Discussion}

The distribution of TFF1 levels in our study (Figure 1), in addition to a wide range of protein levels, implies that breast cancer is a heterogeneous disease. Our finding that high TFF1 levels were related to premenopausal patients is in accordance with the findings of other studies [21,41], although some opposite findings were also reported [32]. Considering that middle-aged group (45-59 years old) consisted of both premenopausal and postmenopausal patients while all patients younger than 45 were premenopausal and all patients older than 59 were postmenopausal in our study, age of the patients was not analyzed as a continuous variable, but stratified into three age subgroups. Although such stratification might be a possible reason why a significant difference in TFF1 levels was not found in relation to age of patients in our study, our results confirm some literature data showing that expression of some ER-inducible proteins, including TFF1, are not significantly related with the age at diagnosis $[42,43]$. Our results also confirm the results of some previous studies reporting that TFF1 expression is negatively associated with histological grade of tumor [21, 30, 32, 44] while positively associated with ER [21, 29, 45] or PR positivity [21, 30, 46]. Considering that a negative correlation between histological grade and ER positivity of tumors has been demonstrated previously [47], results of our study are not surprising. However, not all the studies have demonstrated such association between TFF1 expression and ER [48] or PR positivity [49] or histological grade [29, 32] of tumor. The majority of studies, including ours, failed to demonstrate any association between TFF1 and lymph node involvement or tumor size [21, 30, 32, 50] which may indicate that TFF1 expression is not related to the stage of tumor development. However, a negative association between TFF1 expression and lymph node involvement [27, 44] or tumor size [44] has been reported in some studies.

The choice of an optimal TFF1 cut-off value may be essential for assessing a potential clinical relevance of TFF1 in breast cancer as it may lead to a better stratification of breast cancer patients' subgroups. The result of such stratification should contribute to the improvement of patients' treatment in a way to apply treatment at an earlier stage of the disease or to avoid unnecessary risk for patients who will not benefit of an additional treatment.

In many studies, various statistically-related values were used as cut-off values in order to discriminate between TFF1-positive and TFF1-negative patients that might be at different (low/high) risk for developing distant metastases after primary therapy. In our study, we applied a different approach aiming to find a biologically-related cut-off value that would allow us to discriminate between estrogen-dependent and estrogen-independent TFF1 expression in breast carcinoma. As we were unable to determine such a cut-off value just on the basis of TFF1 level distribution in relation to either menopausal status, histological grade, ER status or PR status, we performed additional analysis of the distribution of TFF1 levels in relation to ER and/or PR status among patients bearing tumors of different histological grade. The additional analysis was performed both within the group of postmenopausal (Figure 2) and within the 
group of premenopausal patients (data not shown). Within the group of postmenopausal patients, the analyses of TFF1 levels revealed that:

- ER-negative patients had significantly lower protein levels in comparison to ER-positive ones, regardless of histological grade of tumor,

- the range of TFF1 levels for ER-negative patients was not comparable with the one corresponding to ER-positive patients, regardless of histological grade of tumor,

- the highest TFF1 level of ER-negative patients was less than $14 \mathrm{ng} / \mathrm{mg}$ protein.

Among premenopausal patients, however, TFF1 levels in ER-negative tumors were neither significantly different from the corresponding levels in ER-positive tumors nor lower than $14 \mathrm{ng} / \mathrm{mg}$, within all three histological grades.

It is well known that postmenopausal women have significantly lower estrogen levels in comparison to premenopausal ones. Nevertheless, these low estrogen levels in postmenopausal women are still able to induce estrogenic response in a way to stimulate TFF1 expression in breast cancer cells. In addition, TFF1 expression in breast cancer may also be induced by growth factors, such as IGF-I [15, 25], via signaling pathway that are related or not to ER, thus making it estrogen-independent. On the basis of our results, we assumed that TFF1 expression is predominantly regulated by growth factors in ER-negative tumors of postmenopausal patients while additionally regulated through indirect estrogen-dependent ER signaling pathways in ER-positive tumors of these patients [51]. We further assumed that indirect estrogen-dependent ER signaling pathways may dominantly regulate TFF1 transcription in ER-negative tumors of premenopausal patients while a classical, estrogen-dependent ER signaling pathway is supposed to be dominant in ER-positive tumors of these patients. Based on these assumptions, the highest TFF1 level in ER-negative tumors of postmenopausal patients was considered as a maximum of estrogen-independent TFF1 expression. A value of $14 \mathrm{ng} / \mathrm{mg}$ of protein was established as the cut-off value to distinguish between estrogen-dependent and estrogen-independent TFF1 expression. Accordingly, patients with TFF1 levels lower or higher than the cut-off value were regarded as those with TFF1-positive or TFF1-negative status, respectively.

It should be noted that PR levels were significantly correlated to TFF1 levels in our study but they seem to have no effect on estrogen-(in)dependent TFF1 expression in breast carcinoma. It may be assumed that PR and TFF1 participate in ER signaling in breast cancer not through common but distinct pathways. Some differences in clinico-pathological and biological characteristics between PR and TFF1 have already been considered by other investigators hypothesizing that estrogen-independent signaling of TFF1 is different from the one of PR in post-menopausal women with ER-positive breast cancer [27]. A question arises on whether a similar difference in TFF1 and PR signaling pathways in breast cancer may be hypothesized in case of estrogen-dependent signaling of TFF1 and we believe it may be.

Detection of low TFF1 expression in normal breast tissue and its high expression in majority of breast carcinomas has been the rationale for numerous studies on the potential clinical value of TFF1 expression in breast cancer [52]. Despite the increasing amount of data on this subject, the findings are inconclusive. In many studies, especially in those using cytosol-based assay, a better prognosis was associated with patients bearing TFF1-positive tumors. A favorable outcome was related to both disease-free and overall survival. Numerous studies also suggested that TFF1 expression may be a predictive indicator of positive response to endocrine therapy in ER-positive breast cancer patients and, moreover, an indicator of sensitivity to AI over Tamoxifen. TFF1 expression has even been identified as an informative marker for the detection of micrometastases [53]. In several studies, however, the prognostic or predictive value of TFF1 expression in breast cancer has not been demonstrated $[27,30]$.

Studies on the clinical value of TFF1 in breast cancer may differ among each other in many issues. Cohorts of patients, the length and end-points of follow-up, methodologies used for determination of TFF1 expression, cut-off values for TFF1 positivity, etc. represent some of the variables that may affect the findings as well as the conclusions of such studies. The follow-up after the surgical therapy is not usually a predefined period of time and differences in patients' follow-up exist within as well as among studies, even when the same end-points are considered. In order to avoid the possible adverse impact of the varying follow-up, a requirement of an equal follow-up period ( 3 years) has been set up for all patients included in the study.

It has previously been reported that a clinical relevance of a biomarker may change in time following the surgical treatment. Several studies reported on the potential of ER status as a prognostic indicator in the early follow-up (mostly 2-2.5 years after the surgery), as its prognostic strength weakened over time $[54,55]$, while others reported on prognostic relevance of ER status in the late follow-up [56]. A time-varying effect of nodal status, tumor size and histological grade on prognosis of breast cancer patients has also 
been reported by several studies [54, 57]. Considering potentially time-dependent clinical relevance of a biomarker, the choice of an optimal follow-up seems important in order to obtain a relevant evaluation of an indicator of the clinical outcome.

The emergence of distant metastases in breast cancer has been shown to follow a double-peak pattern with an early peak at about 2-3 years after surgery and a late peak at about 5 years after surgery [55, 58]. It has been proposed that the surgical therapy induce an increase of probability for the transition of non-dividing single cells to a state of avascular micrometastases [59] and, further, to a phase of vascularization and further growth [60], resulting eventually in an early peak. It is well known that, in terms of breast cancer-specific overall survival, the shorter the interval, the shorter the patient is expected to survive. Given the importance of the early follow-up and by considering a possible time-dependent clinical relevance of a biomarker, it seemed reasonable to us to assess the potential clinical value of TFF1 in primary operable breast carcinoma during the first 3 years of follow-up. Such investigation was also based on our finding that no difference in DFI probabilities was found between groups of patients who were subjected to different modalities of adjuvant systemic therapy: no therapy vs. chemotherapy vs. hormonotherapy vs. chemo+hormonotherapy (data not shown). Our principal aim was to eventually identify subgroups of primary operable breast carcinoma where TFF1 expression might help to discriminate between patients at low vs. high risk for development of distant metastases in a 3-year follow-up after the surgery.

Some previous studies have suggested that high expression of TFF1 is associated with breast carcinomas of a more benign course [27, 32, 44]. Our results are, partially, in contrast with such finding and suggest a possible adverse effect of high levels of TFF1 in breast carcinoma (at least in some of its specific subgroups). It has been presumed that TFF1 might act as a mitogen but early studies failed to demonstrate such biological function. Numerous studies have reported on a role of TFF1 in stimulating the motility and invasion of breast cancer cells [17, 19, 21]. By acting as a motogen, TFF1 was suggested to promote cell dissemination and development of metastases in breast cancer, two processes associated with more aggressive tumor behavior.

A positive role of TFF1 in breast cancer cell migration might provide a rationale for our result, showing a worse outcome for TFF1-positive premenopausal patients bearing ER-positive and PR-positive tumors of histological grade II, in comparison to these patients with negative TFF1 status (Figure 3). As previously mentioned, it may be as- sumed that TFF1 exerts its function through a classical ER signaling pathway that might be predominant in tumors of premenopausal patients due to high levels of estrogen. Additional reason for a worse outcome of these TFF1-positive patients may be a potential role of TFF1 in proliferation of breast cancer cells since increased levels of TFF1 have been demonstrated to stimulate this process in breast cancer [17]. A potential role of TFF1 in proliferation and/or migration of breast cancer cells might be further indicated by the result showing that none of these TFF1-negative patients developed distant metastases during a 3-year follow-up. This result indicates that TFF1 might significantly affect migration and/or proliferation when its concentration is high enough, i.e. at levels higher than the cut-off value.

Menopausal status seemed to be important in terms of clinical outcome among patients bearing ER+, PR+ and TFF1+ tumors of histological grade II. We found a significant difference in DFI probabilities between premenopausal and postmenopausal patients within the indicated phenotype where none of postmenopausal patients developed distant metastases during the first 3 years after the surgery (Figure 4). Our result concerning postmenopausal patients suggests that the proliferation and/or migration of breast cancer cells in ER+, PR+ and TFF1+ tumors of grade II are either stimulated to a low extent or not stimulated at all by higher levels of TFF1. The reason for potentially low/no stimulation of proliferation and/or migration of breast cancer cells might be due to TFF1 signaling that is presumable not directly dependent on estrogen action in these tumors. It should be emphasized that patients with favorable course of the disease, as presented in Figures 3 and 4, have had higher DFI probability than $\mathrm{pN} 0$ patients, for whom DFI probability was 0.86 , while patients with unfavorable course of the disease have had lower DFI probability compared to $\mathrm{pN}+$ patients, for whom DFI probability was 0.68 .

Estrogen-independent TFF1 signaling may be considered as responsible for a potential role of PR in suppressing tumor cell motility and/or proliferation in ER-positive, histological grade II tumors of postmenopausal patients (Figure 5). Expression pathways of TFF1 and PR in breast cancer may be distinct as it was observed that growth factors, such as IGF-I, may increase TFF1 expression while decreasing PR levels, irrespective of ER levels [61]. However, the role of ER (ERa precisely) in the regulation of TFF1 expression can be overridden due to high levels of activating histone modifications in the genome. It has been reported that accumulation of these modifications at TFF1 promoter may facilitate the binding of other transcription factors, rather than ER, that could con- 
tribute to pathways responsible for the development of estrogen-independent breast tumors [62]. Therefore, within the group of ER-positive, postmenopausal patients bearing tumors of histological grade II, we supposed that growth factor-regulated TFF1 signaling pathways (dependent or not on ER) are dominant in PR-negative tumors. Considering the potential role of TFF1 in promoting cancer cell migration and proliferation [19] [18], the cross-talk between TFF1 and PR signaling pathways may be assumed in case of TFF1-positive, postmenopausal patients bearing tumors of histological grade II. It is possible that high levels of PR suppress tumor cell motility by preventing TFF1 dimerization as TFF1 dimer was shown to be more potent than TFF1 monomer in stimulating breast cancer cell migration [19]. High levels of PR might be also presumed to contribute somehow to a lower tumor cell proliferation. Consequently, the motility/proliferation of tumor cells is probably less suppressed in tumors of PR-negative patients. Some previous studies suggested that dual determination of TFF1 and PR may be unnecessary since estrogen-dependent tumors will express both proteins. Our results are in favor of simultaneous determination of these two proteins in, at least, ER-positive tumors of histological grade II as their joint status might help to identify patients at opposite risk for development of distant metastases during the first 3 years after the surgery.

In regard with above mentioned results, it should be emphasized that analysis revealed a uniform distribution of modalities of adjuvant systemic therapies among analyzed subgroups of patients (data not shown). It is noteworthy that none of 14 TFF1-positive postmenopausal patients (Figures 4 and $5)$, in addition to none of 8 TFF1-negative premenopausal patients (Figure 3), all bearing ER-positive and PR-positive tumors of histological grade II, developed distant metastases during the first 3 years of follow-up. This finding suggests that adjuvant systemic therapy might be omitted in case of these patients. One could argue on numbers of patients analyzed in each subgroup with regard to DFI probabilities. Although numbers are small in absolute terms, two facts should be taken into account when considering those numbers. Patients who were included in DFI analysis were supposed to meet several criteria simultaneously with respect to clinico-pathological features, i.e. patients were stratified according to both menopausal status, histological grade, ER status, PR status and TFF1 status. We believe this was quite a demanding prerequisite for the analysis, leading to small numbers of patients analyzed within each subgroup. One may assume that those numbers would be greater within a much larger group of patients (a thousand or several thousands of patients). Having in mind aforementioned, our viewpoint is that numbers of patients related to each subgroup within DFI analysis are not so small in relative terms and that our results regarding DFI analysis do possess significant statistical power. Altogether, a prospective study on a much larger scale of patients is needed to validate our conclusions.

\section{Conclusions}

In summary, our results show that TFF1 expression is correlated with postmenopausal status, histological grade of tumor, ER and PR status, which is in agreement with the findings of the majority of breast cancer studies on TFF1. Distribution of TFF1 levels between ER-positive and ER-negative postmenopausal patients bearing tumors of different histological grade provided us the basis to establish the cut-off value of $14 \mathrm{ng} / \mathrm{mg}$ for distinguishing estrogen-independent from estrogen-dependent TFF1 expression in breast cancer. Determination of a biologically-related cut-off value for TFF1 expression represents the original contribution of this study to the field of breast cancer research. Our results regarding the clinical value of TFF1 in breast cancer indicate that TFF1 status might be helpful in discriminating patients at different risk for development of distant metastases within a 3-year follow-up. Within specific breast cancer subgroups, TFF1 status might identify patients at high risk for relapse (TFF1+ pre II ER+ PR+, TFF1+ post II ER+ PR-), as well as those at low risk for relapse (TFF1- pre II ER+ PR+, TFF1+ post II $\mathrm{ER}+\mathrm{PR}+$ ) who might not even develop distant metastases during the first 3 years of follow-up. It may be assumed that the expression and action of TFF1 in breast cancer is regulated via different signaling pathways through which it stimulates motility and/or proliferation of tumor cells to a different extent. Further investigations are necessary in order to reveal the mechanism of TFF1 action in breast cancer. Determination of TFF1 status could assist in making decision on administering adjuvant therapy for early breast cancer patients during the first 3 years of follow-up.

\section{List of abbreviations used}

TFF1: Trefoil factor;

IGF: Insulin-like growth factor;

EGF: Epidermal growth factor;

TGF: Transforming growth factor;

ERE: Estrogen-response element;

TRE: TPA-response element;

TPA: 12-0-tetradecanoylphorbol-13-acetate;

ER: Estrogen receptor;

AI: Aromatase inhibitor;

PR: Progesterone receptor; 
DFI: Disease free-interval;

NS: Not significant.

\section{Acknowledgements}

This study was supported by Grant No. 175068: "Molecular biomarkers of breast carcinoma and follow-up-dependent changes of their relevance" and Grant No. 173049: "Molecular determinants for tumor marker design" from the Ministry of Education, Science and Technological Development of Republic of Serbia.

\section{Competing Interests}

The authors have declared that no competing interest exists.

\section{References}

1. Thim L. A new family of growth factor-like peptides. 'Trefoil' disulphide loop structures as a common feature in breast cancer associated peptide (pS2), pancreatic spasmolytic polypeptide (PSP), and frog skin peptides (spasmolysins). FEBS Lett. 1989; 250: 85-90. doi:0014-5793(89)80690-8.

2. Thim L, May FE. Structure of mammalian trefoil factors and functional insights. Cell Mol Life Sci. 2005; 62: 2956-73. doi:10.1007/s00018-005-5484-6.

3. Baker ME. Oestrogen-induced pS2 protein is similar to pancreatic spasmolytic polypeptide and the kringle domain. Biochem J. 1988; 253: 307-9.

4. Rio MC, Bellocq JP, Daniel JY, Tomasetto C, Lathe R, Chenard MP, et al. Breast cancer-associated pS2 protein: synthesis and secretion by normal stomach mucosa. Science. 1988; 241: 705-8.

5. Marchbank T, Westley BR, May FE, Calnan DP, Playford RJ. Dimerization of human pS2 (TFF1) plays a key role in its protective/healing effects. J Pathol. 1998; 185: $153-8$ doi:10.1002/(SICI)1096-9896(199806)185:2<153::AID-PATH87>3.0.CO;2-5.

6. Masiakowski P, Breathnach R, Bloch J, Gannon F, Krust A, Chambon P. Cloning of cDNA sequences of hormone-regulated genes from the MCF-7 human breast cancer cell line. Nucleic Acids Res. 1982; 10: 7895-903.

7. Henry JA, Bennett MK, Piggott NH, Levett DL, May FE, Westley BR. Expression of the pNR-2/pS2 protein in diverse human epithelial tumours. Br J Cancer. 1991; 64: 677-82.

8. Prasad NB, Biankin AV, Fukushima N, Maitra A, Dhara S, Elkahloun AG, et al. Gene expression profiles in pancreatic intraepithelial neoplasia reflect the effects of Hedgehog signaling on pancreatic ductal epithelial cells. Cancer Res. 2005; 65: 1619-26. doi:10.1158/0008-5472.CAN-04-1413.

9. Vestergaard EM, Borre M, Poulsen SS, Nexo E, Torring N. Plasma levels of trefoil factors are increased in patients with advanced prostate cancer. Clin Cancer Res. 2006; 12: 807-12. doi:10.1158/1078-0432.CCR-05-1545.

10. Rodrigues S, Rodrigue CM, Attoub S, Flejou JF, Bruyneel E, Bracke M, et al. Induction of the adenoma-carcinoma progression and Cdc25A-B phosphatases by the trefoil factor TFF1 in human colon epithelial cells. Oncogene. 2006; 25: 6628-36. doi:10.1038/sj.onc.1209665.

11. Nunez AM, Berry M, Imler JL, Chambon P. The 5' flanking region of the pS2 gene contains a complex enhancer region responsive to oestrogens, epidermal growth factor, a tumour promoter (TPA), the c-Ha-ras oncoprotein and the c-jun protein. EMBO J. 1989; 8: 823-9.

12. Chalbos D, Philips A, Galtier F, Rochefort H. Synthetic antiestrogens modulate induction of pS2 and cathepsin-D messenger ribonucleic acid by growth factors and adenosine 3',5'-monophosphate in MCF7 cells. Endocrinology. 1993: 133: 571-6.

13. Dickson RB, Lippman ME. Growth factors in breast cancer. Endocr Rev. 1995; 16: 559-89.

14. Barkhem T, Haldosen LA, Gustafsson JA, Nilsson S. pS2 Gene expression in HepG2 cells: complex regulation through crosstalk between the estrogen receptor alpha, an estrogen-responsive element, and the activator protein 1 response element. Mol Pharmacol. 2002; 61: 1273-83.

15. Espino PS, Li L, He S, Yu J, Davie JR. Chromatin modification of the trefoil factor 1 gene in human breast cancer cells by the Ras/mitogen-activated protein kinase pathway. Cancer Res. 2006; 66: 4610-6. doi:10.1158/0008-5472.CAN-05-4251.

16. Poulsom R, Hanby AM, Lalani EN, Hauser F, Hoffmann W, Stamp GW. Intestinal trefoil factor (TFF 3) and pS2 (TFF 1), but not spasmolytic polypeptide (TFF 2) mRNAs are co-expressed in normal, hyperplastic, and neoplastic human breast epithelium. J Pathol. 1997; 183: 30-8. doi:10.1002/(SICI)1096-9896(199709)183:1<30::AID-PATH1085>3.0.CO;2-K.

17. Amiry N, Kong X, Muniraj N, Kannan N, Grandison PM, Lin J, et al. Trefoil factor-1 (TFF1) enhances oncogenicity of mammary carcinoma cells. Endocrinology. 2009; 150: 4473-83. doi:10.1210/en.2009-0066en.2009-0066.
18. Buache E, Etique N, Alpy F, Stoll I, Muckensturm M, Reina-San-Martin B, et al. Deficiency in trefoil factor 1 (TFF1) increases tumorigenicity of human breast cancer cells and mammary tumor development in TFF1-knockout mice. Oncogene. 2011; 30: 3261-73. doi:10.1038/onc.2011.41 onc201141.

19. Prest SJ, May FE, Westley BR. The estrogen-regulated protein, TFF1, stimulates migration of human breast cancer cells. FASEB J. 2002; 16: 592-4.

20. Henry JA, Nicholson S, Hennessy C, Lennard TW, May FE, Westley BR. Expression of the oestrogen regulated pNR-2 mRNA in human breast cancer: relation to oestrogen receptor mRNA levels and response to tamoxifen therapy. Br J Cancer. 1990; 61: 32-8.

21. Corte MD, Tamargo F, Alvarez A, Rodriguez JC, Vazquez J, Sanchez R, et al. Cytosolic levels of TFF1/pS2 in breast cancer: Their relationship with clinical-pathological parameters and their prognostic significance. Breast Cancer Res Treat. 2006; 96: 63-72. doi:10.1007/s10549-005-9041-7.

22. Foekens JA, Portengen H, Look MP, van Putten WL, Thirion B, Bontenbal M, et al. Relationship of PS2 with response to tamoxifen therapy in patients with recurrent breast cancer. Br J Cancer. 1994; 70: 1217-23.

23. Soubeyran I, Quenel N, Coindre JM, Bonichon F, Durand M, Wafflart J, et al. pS2 protein: a marker improving prediction of response to neoadjuvant tamoxifen in post-menopausal breast cancer patients. Br J Cancer. 1996; 74: 1120-5.

24. Elledge RM, Green S, Pugh R, Allred DC, Clark GM, Hill J, et al. Estrogen receptor (ER) and progesterone receptor $(\mathrm{PgR})$, by ligand-binding assay compared with ER, PgR and pS2, by immuno-histochemistry in predicting response to tamoxifen in metastatic breast cancer: a Southwest Oncology Group Study. Int J Cancer. 2000; 89: 111-7. doi:10.1002/(SICI)1097-0215(20000320)89:2<111::AID-IJC2>3.0.CO;2-W.

25. Baron S, Escande A, Alberola G, Bystricky K, Balaguer P, Richard-Foy H. Estrogen receptor alpha and the activating protein-1 complex cooperate during insulin-like growth factor-I-induced transcriptional activation of the pS2/TFF1 gene. J Biol Chem. 2007; 282: 11732-41. doi:10.1074/jbc.M610079200.

26. Spyratos F, Andrieu C, Hacene K, Chambon P, Rio MC. pS2 and response to adjuvant hormone therapy in primary breast cancer. Br J Cancer. 1994; 69: 394-7.

27. Zhou L, Yan T, Jiang Y, Di G, Shen Z, Shao Z, et al. Prognostic and predictive value of TFF1 for adjuvant endocrine therapy in Chinese women with early ER positive breast cancer: comparing aromatase inhibitors with tamoxifen. Breast. 2011; 20: 15-20. doi:10.1016/j.breast.2010.06.002S0960-9776(10)00152-9.

28. Jansen RL, Hupperets PS, Arends JW, Joosten-Achjanie SR, Volovics A, Hillen $\mathrm{HF}$, et al. pS2 is an independent prognostic factor for post-relapse survival in primary breast cancer. Anticancer Res. 1998; 18: 577-82.

29. Foekens JA, van Putten WL, Portengen $\mathrm{H}$, de Koning HY, Thirion B, Alexieva-Figusch J, et al. Prognostic value of PS2 and cathepsin D in 710 human primary breast tumors: multivariate analysis. J Clin Oncol. 1993; 11: 899-908.

30. Soubeyran I, Wafflart J, Bonichon F, de Mascarel I, Trojani M, Durand M, et al. Immunohistochemical determination of pS2 in invasive breast carcinomas: a study on 942 cases. Breast Cancer Res Treat. 1995; 34: 119-28.

31. Gion M, Mione R, Dittadi R, Romanelli M, Pappagallo L, Capitanio G, et al. Relationship between cathepsin $\mathrm{D}$ and other pathological and biological parameters in 1752 patients with primary breast cancer. Eur J Cancer. 1995; 31A: 671-7.

32. Surowiak P, Materna V, Gyorffy B, Matkowski R, Wojnar A, Maciejczyk A, et al. Multivariate analysis of oestrogen receptor alpha, pS2, metallothionein and CD24 expression in invasive breast cancers. Br J Cancer. 2006; 95: 339-46. doi:10.1038/sj.bjc.6603254

33. Luqmani YA, Ricketts D, Ryall G, Turnbull L, Law M, Coombes RC. Prediction of response to endocrine therapy in breast cancer using immunocytochemical assays for pS2, oestrogen receptor and progesterone receptor. Int J Cancer. 1993; 54: 619-23.

34. International Union Against Cancer (UICC). TNM Classification of Malignant tumors, 4th ed. Berlin, Germany: Springer-Verlag; 1987.

35. Scarff RW, Torloni H. Histological typing of breast tumors. International histological classification of tumours, No 2. Geneva: World Health Organization; 1968 ; $13-20$.

36. Bloom HJ, Richardson WW. Histological grading and prognosis in breast cancer; a study of 1409 cases of which 359 have been followed for 15 years. Br J Cancer. 1957; 11: 359-77.

37. Revision of the standards for the assessment of hormone receptors in human breast cancer; report of the second E.O.R.T.C. Workshop, held on 16-17 March, 1979, in the Netherlands Cancer Institute. Eur J Cancer. 1980; 16: 1513-5.

38. Romain S, Laine Bidron C, Martin PM, Magdelenat H. Steroid receptor distribution in 47,892 breast cancers. A collaborative study of 7 European laboratories. The EORTC Receptor Study Group. Eur J Cancer. 1995; 31A: 411-7.

39. EORTC: Manual for clinical research in breast cancer. Lueven, Belgium: EORTC Breast Cancer Cooperative Group; 1991.

40. Lowry OH, Rosebrough NI, Farr AL, Randall RJ. Protein measurement with the Folin phenol reagent. J Biol Chem. 1951; 193: 265-75.

41. Pujol P, Daures JP, Brouillet JP, Maudelonde T, Rochefort H, Grenier J. Time at surgery during menstrual cycle and menopause affects pS2 but not cathepsin $\mathrm{D}$ levels in breast cancer. Br J Cancer. 1999; 79: 909-14. doi:10.1038/sj.bjc.6690145. 
42. Eppenberger-Castori S, Moore DH, Jr., Thor AD, Edgerton SM, Kueng W, Eppenberger U, et al. Age-associated biomarker profiles of human breast cancer. Int J Biochem Cell Biol. 2002; 34: 1318-30. doi:S1357272502000523.

43. Quong J, Eppenberger-Castori S, Moore D, 3rd, Scott GK, Birrer MJ, Kueng W, et al. Age-dependent changes in breast cancer hormone receptors and oxidant stress markers. Breast Cancer Res Treat. 2002; 76: 221-36.

44. Ioachim E, Tsanou E, Briasoulis E, Batsis C, Karavasilis V, Charchanti A, et al. Clinicopathological study of the expression of hsp27, pS2, cathepsin D and metallothionein in primary invasive breast cancer. Breast. 2003; 12: 111-9. doi:S0960977602002904.

45. Henry JA, Piggott NH, Mallick UK, Nicholson S, Farndon JR, Westley BR, et al. pNR-2/pS2 immunohistochemical staining in breast cancer: correlation with prognostic factors and endocrine response. Br J Cancer. 1991; 63: 615-22.

46. Detre S, King N, Salter J, MacLennan K, McKinna JA, Dowsett $M$ Immunohistochemical and biochemical analysis of the oestrogen regulated protein pS2, and its relation with oestrogen receptor and progesterone receptor in breast cancer. J Clin Pathol. 1994; 47: 240-4.

47. Coradini D, Pellizzaro C, Veneroni S, Ventura L, Daidone MG. Infiltrating ductal and lobular breast carcinomas are characterised by different interrelationships among markers related to angiogenesis and hormone dependence. Br J Cancer. 2002; 87: 1105-11. doi:10.1038/sj.bjc.6600556.

48. Angelidou E, Politi E, Sotiropoulou G, Poulianou E, Koutselini H. Evaluation of ER, PR, MIB-1, pS2, and nuclear grade in FNA specimens of cT1 breast carcinomas: clinicopathological correlation. Diagn Cytopathol. 2006; 34: 547-52. doi:10.1002/dc.20510.

49. Pallud C, Le Doussal V, Pichon MF, Prud'homme JF, Hacene K, Milgrom E. Immunohistochemistry of pS2 in normal human breast and in various histological forms of breast tumours. Histopathology. 1993; 23: 249-56.

50. Racca S, Conti G, Pietribiasi F, Stramignoni D, Tampellini M, Valetto MR, et al. Correlation between pS2 protein positivity, steroid receptor status and other prognostic factors in breast cancer. Int J Biol Markers. 1995; 10: 87-93.

51. Heldring N, Pike A, Andersson S, Matthews J, Cheng G, Hartman J, et al. Estrogen receptors: how do they signal and what are their targets. Physiol Rev. 2007; 87: 905-31. doi:10.1152/physrev.00026.2006.

52. Perry JK, Kannan N, Grandison PM, Mitchell MD, Lobie PE. Are trefoil factors oncogenic? Trends Endocrinol Metab. 2008; 19: 74-81. doi:10.1016/j.tem.2007.10.003.

53. Mikhitarian K, Gillanders WE, Almeida JS, Hebert Martin R, Varela JC, Metcalf JS, et al. An innovative microarray strategy identities informative molecular markers for the detection of micrometastatic breast cancer. Clin Cancer Res. 2005; 11: 3697-704. doi:10.1158/1078-0432.CCR-04-2164.

54. Hilsenbeck SG, Ravdin PM, de Moor CA, Chamness GC, Osborne CK, Clark GM. Time-dependence of hazard ratios for prognostic factors in primary breast cancer. Breast Cancer Res Treat. 1998; 52: 227-37.

55. Hess KR, Pusztai L, Buzdar AU, Hortobagyi GN. Estrogen receptors and distinct patterns of breast cancer relapse. Breast Cancer Res Treat. 2003; 78: 105-18.

56. Coradini D, Daidone MG, Boracchi P, Biganzoli E, Oriana S, Bresciani G, et al. Time-dependent relevance of steroid receptors in breast cancer. J Clin Oncol. 2000; $18: 2702-9$

57. Natarajan L, Pu M, Parker BA, Thomson CA, Caan BJ, Flatt SW, et al. Time-varying effects of prognostic factors associated with disease-free survival in breast cancer. Am J Epidemiol. 2009; 169: 1463-70. doi:10.1093/aje/kwp077.

58. Demicheli R, Miceli R, Moliterni A, Zambetti M, Hrushesky WJ, Retsky MW, et al. Breast cancer recurrence dynamics following adjuvant CMF is consistent with tumor dormancy and mastectomy-driven acceleration of the metastatic process. Ann Oncol. 2005; 16: 1449-57. doi:10.1093/annonc/mdi280.

59. Fisher B, Gunduz N, Coyle I, Rudock C, Saffer E. Presence of a growth-stimulating factor in serum following primary tumor removal in mice. Cancer Res. 1989; 49: 1996-2001.

60. Folkman J. Angiogenesis in cancer, vascular, rheumatoid and other disease. Nat Med. 1995; 1: 27-31.

61. Cui X, Schiff R, Arpino G, Osborne CK, Lee AV. Biology of progesterone receptor loss in breast cancer and its implications for endocrine therapy. J Clin Oncol. 2005; 23: 7721-35. doi:10.1200/JCO.2005.09.004

62. Oduro AK, Fritsch MK, Murdoch FE. Chromatin context dominates estrogen regulation of pS2 gene expression. Exp Cell Res. 2008; 314: 2796-810. doi:10.1016/j.yexcr.2008.07.006S0014-4827(08)00271-1. 